\title{
Role of green chemistry in pharmaceutical industry: a review
}

\author{
Ripudaman M Singh ${ }^{1}$, Riya Pramanik ${ }^{2 *}$, Subhajit Hazra ${ }^{3}$
}

1. Assosiate Professor, University Institute of Pharmaceutical Sciences, Chandigarh University, NH-95, Ludhiana - Chandigarh State Hwy, Punjab 140413.

2. Student (Bachelor of Pharmacy), Head, University Institute of Pharmaceutical Sciences, Chandigarh University, NH-95, Ludhiana - Chandigarh State Hwy, Punjab 140413,

3. Research Scholar, University Institute of Pharmaceutical Sciences,

Chandigarh University, NH-95, Ludhiana - Chandigarh State Hwy, Punjab 140413

*Corresponding Author: Riya Paramanik

*Email ID: riyapramanik.2001jan@ gmail.com

\begin{abstract}
Sustainability is the ability to nurture or support a process for an extended period without compromising the needs of the future generation. Sustainable chemistry is a term that refers to the creation of chemical products and processes that decreases or remove the use and production of hazardous substances. Even though sustainable and green technologies have evolved in other scientific fields, their use in the pharmaceutical industry is still initial stage. Therefore, we need to work in green chemistry to ensure its growth in the pharmaceutical industry. Thus, the current review aimed to highlight the need for green chemistry or sustainable chemistry and its principles and its application in the pharmaceutical industry to practice environment-friendly production of pharmaceutical products and reduce or stop the production of harmful intermediates and products during the synthesis process.
\end{abstract}

Keywords: Green Chemistry, Green synthesis, Sustainable Chemistry, Pharmaceuticals, Anastas 12 principles.

\section{Introduction}

Green chemistry is also known as sustainable chemistry, clean chemistry, or benign chemistry. The pharmaceutical industry and other industries are developing very rapidly, which is helping in the fast growth and positive evolution of the medicine and health care sectors. ${ }^{1}$ This, as a result, is reducing deaths and suffering. But what is the point of development when it is also the cause of our environment's deterioration? During the manufacture of chemicals, the different steps associated with the synthesis process generate many pollutants, intermediates, and other waste products. These unwanted waste products are released into the environment without proper purification. ${ }^{2}$ This lead to an enhancement in the level of pollutants in our environment and hence causes its deterioration. As a result, people are increasingly becoming aware and trying to move towards 'green Chemistry' to protect their surroundings. ${ }^{1}$

Green or sustainable chemistry is a term related to creating chemical products and processes that decrease or stop the use and production of hazardous substances. As a new branch of chemistry with ecological approaches, it includes reducing or eliminating the use of harmful substances in chemical processes as well as reducing harmful and toxic intermediates and products. ${ }^{2}$

\section{Brief History}


In the 1960s, the publication of the book 'Silent Spring' opened the eyes of many. The scientific book raised awareness about ecological perception and has shown the risks associated with the over-use of natural resources. The book described how certain chemicals are affecting our ecosystem. ${ }^{4}$

The EPA or Environmental Protection Agency was launched in 1970. The Stockholm conference was conducted in 1972 in Sweden, which was attended by many countries along with the members of the United Nations. In this conference, the members discussed the environmental damages that cause the depletion of the ecosystem and alerted everyone regarding the same. ${ }^{4}$

Till the 1980s, the chemical industry and the EPA concentrated mainly on pollution and harmful toxins. However, a significant shift occurred among chemists as scientists started spreading environmental awareness and researching avenues to prevent pollution. ${ }^{4}$

The Organization for Economic Co-operation and Development (OECD), an international body consisting of about 30 industrialized countries, held meetings and made recommendations based on a cooperative change in existing chemical synthesis processes and the prevention of pollution. ${ }^{4}$

In 1999, Paul Anastas published a paper that talked about the importance of green chemistry and postulated 12 principles of green chemistry. ${ }^{1}$

\section{Study Methodology}

\subsection{Aim of the review}

The current review aimed to highlight the 12 principles of green chemistry and its use in the pharmaceutical sector.

\subsection{Objective of the review}

To understand and provide an elaborate discussion on the importance of green synthesis in pharmaceutical sector.

\subsection{Material and method}

\subsubsection{Search strategy}

To carry out this review we searched out the articles from various databases which includes PubMed and scopus. All the papers that discussed about green synthesis were screened. Furthermore, the keywords included: green synthesis, green chemistry, principles of green chemistry, green synthesis in pharmaceuticals industry, application of green chemistry.

\subsubsection{Study selection criteria}

Inclusion criteria: Publication describing about green chemistry, its history and applications were included in the review.

Exclusion criteria: Publication were excluded from consideration for the review, if they did not met the inclusion criteria.

\section{Discussion of Review}

\section{Twelve principles of green chemistry}

Green chemistry is the branch of chemistry in which we design chemical substances without causing any harm to the environment. The 12 principles of green chemistry were postulated by Paul Anastas and John Warner.

The principles of green chemistry explain the reduction and the removal of dangerous, harmful, or hazardous substances which are formed during the synthesis and the production process. ${ }^{6}$ 


\section{Prevention}

The first principle of green chemistry is based on the principle of prevention, which is further based on the fact that "It is better to prevent waste than to treat or pack up waste after it's already formed". A lot of waste material is being generated during the whole synthesis process in the industries. These waste materials are released into the water bodies without proper treatment, as a result of which, the environment is getting polluted. Now the question arises, what exactly is waste? "Waste is the substance that's not incorporated into the ultimate material during manufacture, or a product that don't serve a requirement and excessive resource use".

Completely omitting the generation of waste product in the pharmaceutical industry is not possible, hence the only thing that can be done is either to reduce amount of solvent, or to replace one solvent with another to decrease the amount or number of solvents used during the final synthesis process. For example, in the new "green synthesis" process of sertraline, methanol is used as the sole solvent.

Measure of waste can be described by the following.

$$
\text { Environmental factor }(E)=\frac{\mathrm{kg} \text { waste }}{\mathrm{kg} \text { product }}
$$

E factor was described by Roger Sheldon. It is the ratio of weight of waste with the weight of product produced during the synthesis process.

$$
\text { PMI (Process mass intensity) }=\frac{\text { quality of raw material input }(\mathrm{kg})}{\text { quality of bulk API product out }(\mathrm{kg})}
$$

PMI is the criteria favoured by ACS Green Chemistry Institute Pharmaceutical Roundtable (ACS GCIPR) which is the ratio of quantity of raw material used to the quantity of bulk API produced during the manufacturing process. ${ }^{1}$

\section{Atom economy}

"Synthetic methods should be designed to maximise incorporation of all materials utilized in the method into the ultimate product".

Atom economy is the measure of the amount of atoms present in the final product after the end of chemical reaction/ synthesis from the starting material. Atom economy was developed by Berry Trost of Stanford university in the year 1991. The intermediates which are formed during the reaction and don not have any use decreases the atom economy and increases waste. Atom economy is considered more efficient than percentage yield of the reaction. ${ }^{1}$

Hence, the chemical synthesis should be done in such a way so as to get the maximum amount of the finale product. ${ }^{1}$

$$
\% \text { atomic economy }=\frac{\text { weight of the desired product }}{\text { weight of all reactants }} *(100)
$$

\section{Less hazardous chemical synthesis}

"Wherever practicable, synthetic methods should be designed to use and generate substances that possess little or no toxicity to human health and the environment."1

Most of the chemical synthesis reactions, which take place in many stages, use harmful reagents. Even though the product does not contain these toxic substances, there is a risk of its contamination and redesigning these processes is a task of green chemistry. ${ }^{1}$ Hence, this principle is based on design of a process of synthesis which would produce minimum or lowest possible toxic product. The main aim is to avoid the use hazardous chemicals as the starting point if the safer available. $^{1}$ 


\section{Designing safer chemicals}

"Chemical products should be designed to effect their desired function while minimizing their toxicity".

The designing of the chemicals requires the knowledge of its structure. One of the biggest challenges in designing a safe chemical is maintain the basic function of the chemicals and its effectiveness, while reducing it toxicity. In other words, the use of hazardous or harmful chemicals should be eliminated whenever possible, keeping in mind that the efficacy of the same has been maintained. ${ }^{1}$ This principle is used in the development of new chemicals, in order to maintain its potential by limiting the use of toxic solvents or substances.

\section{Safer solvents and auxiliaries}

"The use of auxiliary substances (e.g., solvents, separation agents, etc.) should be made needless wherever possible and harmless when used."

Most of the chemical reaction uses solvent or any other reagent to carry out the ongoing reaction. These solvents or auxiliaries used might produce toxicity or act as a hazardous substance. The use of solvents during reaction is necessary and can-not be avoided but they should be chosen in such a way that they decrease the total heat needed during the synthesis reaction an should have minimum toxicity. ${ }^{1}$

\section{Design for energy efficiency}

"Energy requirements should be recognized for their environmental and economic impacts and should be minimized. Synthetic methods should be conducted at ambient temperature and pressure".

This principle suggests that the reaction has to be designed in such a manner to that it requires minimum amount of energy during the reaction process.

We can decrease the amount of energy used during the synthesis process by carrying out the reaction process at low temperature and pressure. ${ }^{1}$

\section{Use of renewable feedstock}

"A raw material or feedstock should be renewable rather than depleting whenever technically and economically practicable"1

\section{Reduce derivatives}

"Unwanted derivatization should be decreased or eliminated, if possible, because such steps require additional reagents and can generate waste."

One of the important point in green chemistry is to synthesize the desired product without the use of chemical derivatives. The use of derivatives should be minimized, as it produces unwanted waste. $^{1}$

\section{Catalysis}

"Catalytic reagents (as selective as possible) are superior to stoichiometric reagents".

This principle promotes the utility of biodegradable catalysts, which consumes less energy and protects the environment. A reaction with high atom economy can be produced using catalyst. The catalyst can be reused many times as it is not used up in the reaction and do not produce waste. ${ }^{1}$

\section{Design for degradation}

"Chemical products should be designed so that at the end of their function they break down into harmless degradation products and do not remain in the nature"

This principle suggests that chemical products should be designed in such a way that once it has been utilized, it should breakdown into particles which do not produces any toxic effect in our environment. ${ }^{1}$ 


\section{Real time pollution prevention}

"Analytical methodologies need to be further developed to allow for real-time, in-process monitoring and control prior to the formation of hazardous substances".

Chemical reactions should be monitored when it is occurring. This can prevent release of toxic by-product and can prevent the occurrence of fatal interactions as reaction can be stopped immediately while monitoring. ${ }^{1}$

\section{Safer chemistry for accident prevention}

"Substances and the form of a substance used in a chemical synthesis should be chosen to reduce the probability of chemical accidents, including releases, explosions, and fires".

Risk is always present while handling chemicals. This principle highlights the importance of safe handling handling of chemicals, so as to avoid unnecessary accidents. Accidents can be avoided by taking proper measures during the chemical reaction. ${ }^{1}$

\section{Green chemistry in industry}

\section{Nanoparticles}

Nanoparticles are the particles whose size ranges between $1 \mathrm{~nm}$ to $100 \mathrm{~nm}$. Nanoparticles have enhanced properties due to their large surface area. The actual method used for the synthesis of nanoparticles is toxic and hazardous for the environment. The drawback of traditional synthesis method is that, the colloidal solution gets contaminated due to the presence of by-products. Hence to overcome this issue green synthesis of nanoparticles was introduced. These nanoparticles are not only eco-friendly but also cost effective and can be used for the large-scale production. ${ }^{5}$ This follows some principles of green chemistry such as prevention, less hazardous chemical synthesis, designing safer chemicals and real time prevention of pollution. In the field of pharmacy, nanotechnology is in its developing stage. ${ }^{6}$ Earlier physical and chemical methods were employed for the synthesis of nanoparticles. Increased demands of nanoparticles have led to their large-scale production. Hence, the commercial method was developed to synthesize metal nanoparticles. However, the use of toxic solvents or high energy in these methods has led to a growing awareness regarding the need of using clean, nontoxic and environmental-friendly methods to synthesize nanoparticles. ${ }^{7}$ Green synthesized nanoparticles are more biocompatible than chemically synthesized ones. The three major benefits of using green synthesized nanoparticles are. ${ }^{8}$
a) Environment friendly ${ }^{8}$
b) Non-toxic ${ }^{8}$
c) Cheap $^{8}$

Many microorganisms like yeast, fungi, bacteria, plants, etc can be used for the synthesis of nanoparticles. ${ }^{9}$ Table. 1 shows some examples of microorganisms which are used for green synthesis of nanoparticles.

Table 1: Examples of microorganisms which are used for green synthesis of nanoparticles

\begin{tabular}{|l|l|}
\hline \multicolumn{1}{|c|}{ Source } & \multicolumn{1}{|c|}{ Examples } \\
\hline Yeast & $\begin{array}{l}\text { Rhodosporidium diobovatum }{ }^{10} \text {, Saccharomyces boullardii } \\
{ }^{11}, \text { etc. }\end{array}$ \\
& \\
\hline
\end{tabular}




\begin{tabular}{|l|l|}
\hline Fungi & Aspergillus fumigatus, Aspergillus clavatus, ${ }^{12}$ etc. \\
\hline Bacteria & ${\text { Serratia } \text { sp }^{13}}^{13}$ \\
\hline Plant & Melia azedarach $^{14}$, Tridax procumbens $^{5}$, etc. \\
& \\
\hline
\end{tabular}

\section{Green solvents}

Green solvents are the solvents that are used instead of conventional solvents. In their 12 Principles of Green Chemistry, Anastas and Warner claimed the utilization of "safer solvents and auxiliaries". ${ }^{15}$ In many synthesis processes flammable organic solvents are used for the synthesis process, however, these conventional solvents are harmful and toxic to our environment. Hence green solvents are now replacing the usage of conventional solvents in various industries. ${ }^{16}$

There is excellent sort of solvents. the choice of a correct solvent for the specific reaction may be a key issue to achieve success during a reaction procedure. While selecting a solvent throughout a reaction, these properties ought to be considered:

- Chemical compatibility with reagents and products.

- Solving of reagents.

- Temperature of the method. ${ }^{17}$

Water as solvent: The continuous increase within the requirement of a more sustainable approach in synthesis procedures results in an emerging interest within the use of water as a solvent. The use of water as a solvent in chemical synthesis is one of the best options in green chemistry, to minimize the release of harmful chemicals in the environment. Using water as a solvent, reactions are often performed under mild experiment conditions and therefore the catalysts are often reused, which decreases the general cost of the merchandise. ${ }^{18}$

Ionic liquids: In the context of green solvents, we might wish to discuss ionic liquids (ILs) that, a minimum of for a particular time, are considered as not only designer solvents but also as green solvents, mainly because they need a negligible vapor pressure and intrinsically don't contribute to the volatile organic compounds' problematic. ${ }^{19}$

Glycerol: green technology is that the production of biodiesel and bioethanol. During biodiesel production, an enormous amount of byproduct is generated and wasted i.e. "glycerol". This glycerol has enormous potential for applications in the pharmaceutical, food, and explosive industries. (20)

\section{Antimicrobial bandages}

Bandage is a material which is used to cover up the wounds or injured body parts. It provides support to the wound and the surrounding tissues. This follows the first and twelfth principle of green chemistry. ${ }^{5}$

Green synthesis of nanoparticles is done for making wound healing bandages. The nanoparticles are then impregnated on the bandages. For example, silver nanoparticles were synthesized by using the weed plant Tridax procumbens (impregnated on the bandage), which has shown antimicrobial activity against gram-positive and gram-negative bacteria. ${ }^{5}$

A time-saving, environmentally friendly, and cheap synthesis of silver (Ag) and philosopher's wool $(\mathrm{ZnO})$ nanoparticles was done by using Prosophis fracta and occasional. The minimum 
inhibitory concentrations (MIC) of these silver and $\mathrm{ZnO}$ nanoparticles and mixture thence, $\mathrm{Ag} / \mathrm{ZnO}$, were determined on Acinetobacter baumannii and bacteria genus aeruginosa cultures. Cotton wound bandages were inseminated with nanoparticles of silver and $\mathrm{ZnO}$ and mixed $\mathrm{Ag} / \mathrm{ZnO}$ nanoparticles inside the neighborhood of calculated MIC and their antimicrobial activity was studied in vitro; each kind of nanoparticles showed a high medication activity of bandages. ${ }^{21}$

\section{Green synthesis of drugs}

Green processes are developed to synthesize drugs to prevent release of harmful and toxic byproducts in the environment. Almost all the principles of green chemistry has been applied for the same. Such as prevention, atom economy, less hazardous chemical synthesis, safer solvent, catalysis etc. $^{2}$

\section{CASE STUDY}

\section{Sertraline:}

Sertraline is an anti-depressant drug. sertraline was launched by Pfizer in the year 1991. It shows its pharmacological activity by inhibiting the uptake of serotonin.

In the traditional the synthesis process of sertraline by Friedel craft acylation needed excess of aluminium chloride was needed and the reaction was carried out by using carbon disulphide, which is a harmful solvent. Titanium tetrachloride was used for the condensation reaction in the synthesis process as a result titanium waste was generated and tetrahydrofuran was used as a solvent.

In the green synthesis of sertraline tetrahydrofuran was replaced by ethanol which the increased formation of imine to more than $95 \%$, furthermore titanium tetrachloride $\left(\mathrm{TiCl}_{4}\right)$ is no longer used for imine formation and hence titanium waste is also not generated. ${ }^{2}$

\section{Talampanel}

Talampanel is also known as LY300164. It is a drug which is used for the treatment of neurological disorders such as alzheimer's disease, parkinson's disease, epilepsy and etc. It shows its pharmacological action by targeting the AMPA component.

The actual synthesis process of talampanel consisted of eight steps and had about $8 \%$ yield. This process used hydrazine, borane, chromium trioxide and perchloric acid which were not environment friendly.

Furthermore, the use of hydrazine led to API contamination, which was a major concern along with the use of chromium trioxide which produced large amount of chromium waste during the manufacturing process.

Due to the above-mentioned drawbacks the, synthesis process of talampanel was redesigned. In its green synthesis chromium is replaced by air and hydrazine is replaced by less harmful acetyl hydrazine. Perchloric acid is no longer used in then synthesis process, which is an environment pollutant. The final yield after the greener approach is about $91 \% .^{2}$

\section{Sildenafil citrate}

Sildenafil comes under the category phosphodiesterase inhibitor which shows its pharmacological action by inhibiting phosphodiesterase enzyme. it was developed by Pfizer. It is mainly used in case of erectile dysfunction or impotencey in men. ${ }^{2}$

In the earlier synthesis process of Sildenafil citrate the final yield was about $4.2 \%$ which is not suitable for large scale manufacturing. The chloro-sulfonation step in the synthesis needed chorosulfonic acid in excess amount which resulted in the generation of hazardous waste. Furthermore, this was a large eleven step process, hence shortening the synthesis step was also required. ${ }^{2}$ 
The green approach used the reagent t-butanol and its potassium base. In this process, chlorosulfonation moved to the earlier steps, and cyclization shifted to the later steps.

This allowed the purification of the workups within the steps after the sulfonation to eliminate the harmful remains, which is toxic. The final step is now run at high concentration, which further decreases the waste of solvents. This also increased the yield to $97 \%$ (average yield). ${ }^{2}$

\section{Quinapril}

Quinapril comes under the category of anti-hypertensive agents. It is used for the treatment of hypertension that is to lower blood pressure and congestive heart failure. ${ }^{2}$

Quinapril expresses its therapeutic activity by inhibiting the angiotensin-converting enzyme.

The original manufacturing process includes the use of many undesirable solvents such as, the use of methyl chloride, hydroxy benzotriazole, which is explosive in nature, dicyclohexylcarbodiimide and copious toluene to remove acetic acid during the synthesis process. ${ }^{2}$

During the synthesis of quinapril, intermolecular cyclization occurs, which results in the formation of diketopiperazine impurity. ${ }^{2}$

The green approach focused on the stoppage of acetic acid, which would result in minimizing diketopiperazine formation. This was done by replacing the initial material with the commercially available $\mathrm{N}$-carboxy anhydride. When reacted with the isoquinolinecarboxylic acid t-butyl ester salt, the self-activated anhydride led to direct amide coupling, allowing the elimination of DCC, it's waste product dicyclohexylurea (DCU), and the need for a chlorinated solvent. ${ }^{2}$

\section{CONCLUSION}

Green chemistry is the branch of chemistry that mainly focuses on sustainability and hence is also referred to as sustainable chemistry. Sustainability in green chemistry is attained by either using a natural chemical moiety for the synthesis process or using the chemical synthesis process that causes minimal harm to the environment. The importance of green chemistry and its application in the industry has been reviewed.

\section{ACKNOWLEDGEMENT}

None

\section{REFERENCE}

1) Ivanković A, Dronjić A, et al. Review of 12 Principles of Green Chemistry in Practice. 2017; 6(3): 39-48

2) Berkeley W and Zhang J. Green process chemistry in the pharmaceutical industry. 2009;2(10):193-211

3) Nydia T, Cecile B. Green and sustainable chemistry. 2018;8(3):1-10

4) De Marco B.A, Rechelo B.S, et al. Evolution of green chemistry and its multidimensional impacts: a review. 2019;27(1):1 8

5) Chopade V, Kamble D. Application of green silver nanoparticles synthesized using green extract of Tridax Procumbens for preparation of clinical antimicrobial bandages. 2021;11(1):10-13

6) Gaur A, Jain NK. Advances in green synthesis of nano particles.2019;47(1):844-850.

7) Thununggunta $T$, Reddy AC et al. Green synthesis of nanoparticles: current prospectus.2015;4(4):303-323

8) Monika B, Anupam B, et al. Green synthesis of gold and silver nanoparticles.2015;6(3):1710-1716.

9) Vardhana J, Kathiravan G. Biosynthesis of silver nanoparticles by endophytic Fungi Pestaloptiopsis pauciseta isolated from the leaves of Psidium guajava linn. 2015;31(1):2931.

10) Seshadri S, Sarany K, etal. Green synthesis of lead sulfide nanoparticles by lead resistant marine yeast, Rhodosporium diobovatum. 2011;27(5):1464-1469.

11) Korbekandi $\mathrm{H}$, Jauneghani RM, etal. Synthesis of silver nanoparticles using biotransformations by saccharomyces boulardii. 2014;3(4):271-277.

12) Siddiqui KS, Husen A. Fabrication of metal nanoparticles by fungi and metal salts: scope and applications. 2016;11(1):115.

13) De silva C, Noor AAM, etal. The green synthesis and characterisation of silver nanoparticles from Serratia spp.2020;19(3):1327-1339.

14) Ahemad A, Syed WAA, et al. Green route synthesis of $\mathrm{ZnO}$ nanoparticles mediated by Melia azedarach for microbiological applications. 2020;1(1):1-15.

15) Devi BK, Naraparaju S, etal. Green chemistry and green solvents: An overview. 2020;7(3):314-325.

16) Bubalo MC, Vidovic S, etal. Green solvents for green technologies.2015;90(9):1631-1639. 
17) Clavo Floures FG, Montaegudo Arrebola MJ, etal. Green and biobased solvents.2018;376(3):1-40.

18) Majhi KC, Karfa P. Water as the green solvent in organic chemistry.2019;54:182-201.

19) Katharina H, Werner K. Some aspects of green solvents.2018;21(6):572-580.

20) Kawale HD, Acharaya S. Application of glycerol as green nsolvent.2019;54:87-105.

21) Khatami M,Verma RS, etal. Application of green synthesised $\mathrm{Ag}, \mathrm{ZnO}$ and $\mathrm{Ag} / \mathrm{ZnO}$ nanoparticles for making clinical antimicrobial wound healing bandages.2018;10:9-15.

22) Sahoo T, Panda J, et al. Green solvent> green shadow on chemical synthesis.2020;17(6):426-439.

23) Wegner K, Bernes D, etal. Evaluation of greener solvent for solid phase peptide synthesis.2021;14(1):152-162.

24) Mawrenson SD. Greener solvent for solid phase organic synthesis.2018;90(1):157-165.

25) Molnar M, Loncaric M, etal. Green chemistry approaches to the synthesis of coumarin derivatives.2020;24(1):4-43.

26) Huston M, DeBella M, etal. Green synthesis of nanomaterials.2021;11(8):2130.

27) Gaur A, Jain NK, et al. Advances in green synthesis of nanaoparticles.2019;47(1):844-851.

28) Singh NB, Jain P, et al. Green synthesis and applications of nanomaterial.2021;22(13):1705-1747.

29) Zhu YT, Li ZJ, et al. Applications of lignin derivative for green synthesis.2019;4(3):210-244.

30) Mondal S. Sulfonamide synthesis under green conditions.2020;51(7):1023-1044.

31) Gupta M, Paul S, et al. General aspects of 12 basic principles of green chemistry with application. 2010;99(10):1341-1360.

32) Abdussalam M.W, Ali AQ, et al. Green chemistry: Principle, application and disadvantages. 2020;4(4):408-423

33) Koel M, Kaljurand M, Application of the principle of green chemistry in analytical chemistry. 2006;78(11):1993-2002.

34) Haq N, Iqbal M, et al. Applying green analytical chemistry for rapid analysis of drugs: Adding health to pharmaceutical industry.2012;(10):777-785.

35) Cai W.Q, Cheng B, et al. Developing the green chemistry principle. 2009;21(10):2001-2008 